Electrostatic Control of Dewetting Dynamics

\title{
Electrostatic Control of Dewetting Dynamics
}

Andrew M. J. Edwards, ${ }^{1}$ Rodrigo Ledesma-Aguilar, ${ }^{2}$ Michael I. Newton, ${ }^{1}$ Carl V. Brown, ${ }^{1}$ a) and Glen $\mathrm{McH} \mathrm{H}^{2}$

${ }^{1)}$ SOFT Group, School of Science and Technology, Nottingham Trent University, Clifton Lane, Nottingham NG11 8NS,

$U K$.

${ }^{2)}$ Smart Materials and Surfaces Laboratory, Department of Mathematics, Physics and Electrical Engineering,

Northumbria University, Ellison Place, Newcastle upon Tyne NE1 8ST, UK.

(Dated: 4 June 2020)

The stability of liquid films on surfaces are critically important in microscale patterning and the semiconductor industry. If the film is sufficiently thin it may spontaneously dewet from the surface. The timescale and rate of dewetting depend on the film repellency of the surface and the properties of the liquid. Therefore, control over the repellency requires modifying surface chemistry and liquid properties to obtain the desired rate of film retraction. Here, we report how the dynamics of a receding thin liquid stripe to a spherical cap droplet can be controlled by programming surface repellency through a non-contact electrostatic method. We observe excellent agreement between the expected scaling of the dynamics for a wide range of voltage-selected final contact angles. Our results provide a method of controlling the dynamics of dewetting with high precision and locality relevant to printing and directed templating.

The spontaneous retraction of a thin liquid film on a surface, e.g. dewetting, is a ubiquitous and everyday phenomenon critically important in applications such as printing, coating and micro and nanoscale patterning ${ }^{1}$. For example, in the semiconductor industry surfaces are chemically treated to prevent dewetting of thin resist films and associated growth of dry regions $\mathrm{s}^{2,3}$. Dewetting occurs when it is energetically favourable to remove the liquid film from the surface, a situation controlled by the surface energies of the solid-vapour $\left(\gamma_{S V}\right)$, solid-liquid $\left(\gamma_{S L}\right)$ and liquid-vapour $(\gamma)$ interfaces, and defined by a negative spreading parameter, $S=\gamma_{S V}-\left(\gamma_{S L}+\gamma\right)$. The dynamics of dewetting for a small volume of liquid has been shown to comprise of two distinct regimes ${ }^{4}$. First the formation of a capillary rim around the circumference of the film and second a return to a spherical cap droplet eventually reaching an equilibrium shape which intersects the solid with an equilibrium contact angle, $\theta_{e}$. Studies of capillary rims in the growth of dry holes in thin liquid films suggest that the speed of the contact line remains constant and scales with a cubic power law of the equilibrium contact angle $e^{5,6}$. Control over $\theta_{e}$ and therefore the speed of dewetting is performed by altering surface chemistry or topography ${ }^{7}$.

In recent years electrowetting on dielectric (EWOD) and dielectrowetting have proven popular and versatile methods of electrostatically modifying the contact angle ${ }^{8,9}$, with both methods finding applications in microfluidics ${ }^{10,11}$, displays $^{12,13}$ and optofluidics ${ }^{14,15}$. Electrowetting has previously been used to study the wetting and dewetting dynamics of spherically capped droplets in both liquid-air ${ }^{16}$ and liquidliquid systems ${ }^{17}$ over a range of contact angles. EWOD however cannot be used for thin film dewetting dynamics because of the saturation of the contact angle at high voltages ${ }^{18}$. Dielectrowetting, unlike EWOD does not suffer from contact angle saturation and has been shown to provide complete spreading and super-spreading to produce thin liquid films ${ }^{19,20}$. Dielectrowetting induced thin liquid films have previously been used to study dewetting phenomena by rapid quenching of the

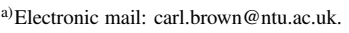

electric field, restoring the film back to the droplet state $e^{4,21}$.

In this work, we report the programming of surface repellency using a non-contact electrostatic method which achieves dewetting to a voltage-selectable final contact angle, $\theta_{R}$ up to any value of $\theta_{e}$. We demonstrate the effect of controlling the value of $\theta_{R}$ for both the capillary rim and spherical cap regimes during dewetting of a thin rectangular film. We show excellent agreement in both regimes with the theoretical predictions of the dynamics of dewetting. Moreover, we show that the pearling instability present in retracting liquid rivulets can be completely suppressed by the electrostatic control ${ }^{22}$.

We pattern indium tin oxide coated glass surfaces with a series of inter-digitated electrodes arranged in a linear stripe array with an electrode with equal width and gap, $d=5 \mu \mathrm{m}$, by standard photolithographic techniques. The electrode pattern is arranged in a rectangular array with length, $l=5 \mathrm{~mm}$ and width, $w=1.25 \mathrm{~mm}$, where the stripes are parallel to the $\mathrm{x}$-direction. Electrodes are capped with a $1 \mu \mathrm{m}$ thick planarizing dielectric layer of SU-8 (Microchem) and a liquid repellent surface coating of Teflon AF (Sigma, $\leq 100 \mathrm{~nm}$ ). Upon application of a sinusoidal AC voltage, $V$, between the electrodes a non-uniform electric field localised in the the vicinity of the surface is induced with a dielectrophoretic energy proportional to the square of the applied voltage ${ }^{23}$. Deposited droplets of non-volatile trimethylolpropane triglycidyl ether (TMP-TG-E) respond by spreading over the solid until they cover an area of the surface such that the overall energy (surface plus dielectrophoretic) is minimized. This increase in area is accompanied by a reversible reduction in the solid-liquid contact angle, $\theta$, with increasing $V$ given by $\cos \theta(V)=\cos \theta_{e}+\alpha V^{2}$, where $\alpha=\pi \varepsilon_{0}\left(\varepsilon_{L}-\varepsilon_{V}\right) / 8 \gamma d$ and $\varepsilon_{0}, \varepsilon_{l}, \varepsilon_{v}$ are the permittivity of free space, liquid and air respectively ${ }^{19,24}$.

A state of complete wetting, i.e. $\theta(V) \approx 0^{\circ}$, is reached for voltages that equal or exceed a threshold voltage, $V_{t h}$, producing a thin spread film of liquid. For our system, using a $10 \mathrm{kHz}$ sine AC voltage we experimentally find $V_{t h}=204 \mathrm{~V}$, all voltages given are the rms values. By application of an applied voltage $\geq V_{t h}$ a thin rectangular film of TMP-TG-E is formed from a spherical cap shaped droplet with a volume, $\Omega$ $=0.44 \mu \mathrm{L}$ (see Fig. 1). Here the thickness of the rectangular 


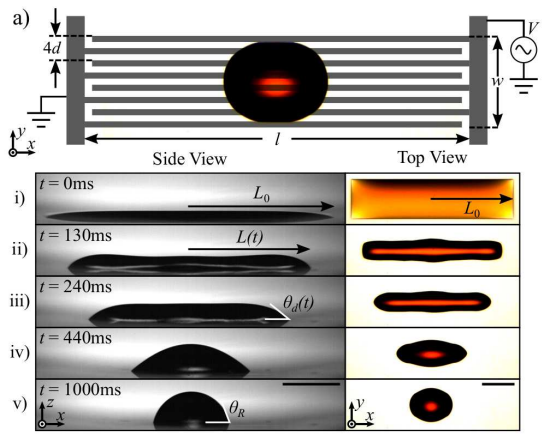
b) $\theta_{R}=74.8^{\circ}$

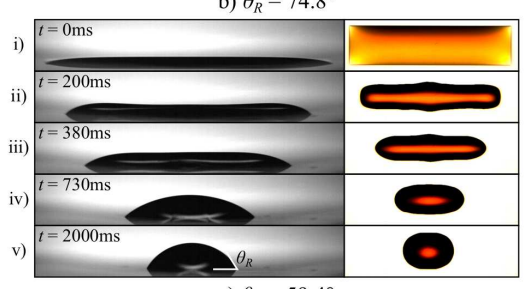
c) $\theta_{R}=59.4^{\circ}$

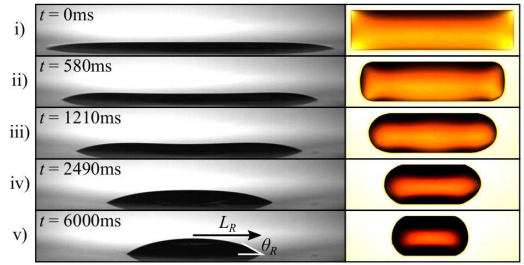

d) $\theta_{R}=30.9^{\circ}$

FIG. 1. a) Schematic of the experimental configuration and underlying electrode structure. Not to scale. b-d) Side and top view images of a dewetting thin rectangular film under biasing retraction voltage, $V_{R}$. a) $V_{R}=0 \mathrm{~V}$. b) $V_{R}=120 \mathrm{~V}$. c) $V_{R}=180 \mathrm{~V}$. i) $L_{x}=1$. ii) $L_{x}=0: 8$ iii) $L_{x}=0: 6$. iv) $L_{x}=0: 2$. v) $L_{x}=0: 003$. Scale bar is $1 \mathrm{~mm}$. Images have been edited for brightness and contrast.

film can be well approximated by $h_{0} \approx \Omega / l w \approx 70 \mu \mathrm{m}$. We define an aspect ratio for our rivulet as, $A R=l / w=4$, for this value of $A R$ we find that axial retraction dominates over droplet pinch-off through the pearling instability.

All experiments were carried out at a constant temperature of $21^{\circ} \mathrm{C}$ at which the physical properties of TMP-TG-E are $\gamma=43.17 \mathrm{mN} / \mathrm{m}, \eta=188.17 \mathrm{mPa} . \mathrm{s}$ and $\rho=1165 \mathrm{~kg} / \mathrm{m}^{3}$. The Bond number is given by $B o=\rho g L^{2} / \gamma \approx 10^{-1}$ indicating that final droplets are not significantly gravitationally distorted. Measurements of the advancing and receding angles for the system were made using the droplet inflation method and found to be $95 \pm 3^{\circ}$ and $81 \pm 3^{\circ}$ respectively.

Here we use dielectrowetting as the method to provide an initial rectangular shaped thin liquid film from a spherical cap droplet using a setup voltage of $210 \mathrm{~V}$. This approach allows repeated studies of the dewetting dynamics from the same initial thin film where crucially we are able to readily program the degree of liquid repellency of the solid surface by application of a constant retraction voltage $V_{R}$. This constant retraction voltage allows voltage-selection of the final contact angle up to and including the equilibrium angle i.e. $\theta_{R} \leq \theta_{e}$, where in this study the final angles are in the range $31^{\circ}<\theta_{R}<$ $76^{\circ}$. Fig. 1 shows side and top view images for an initially rectangular thin film relaxing back to a droplet for three different values of $V_{R}$ (see video $\mathrm{S} 1$ for the complete retraction process). As the final base length and contact angle of the droplet differs with each applied retraction voltage we find it convenient to rescale the data using the dimensionless variable $L_{x}=\left(L(t)-L_{R}\right) /\left(L_{0}-L_{R}\right)$, where $L(t)$ is the instantaneous base length, $L_{0}$ is the initial base length and $L_{R}$ is the voltageselected final base length. This scaling allows us to compare equivalent points in the dewetting dynamics.

Fig. 1 shows that initially there exists a short transient while the rectangular film dewets into a thin liquid rivule ${ }^{25}$. Following this a capillary rim is formed at short timescales and after coalescence of these rims a spherical cap droplet is formed at long timescales ${ }^{4,26}$. Due to the short length scale of the y-direction the dewetting and appearance of capillary rims is more prominent in the $\mathrm{x}$-direction. We find that for $0.3<L_{x} \leq 1$ the droplet is in the capillary rim regime and for $L_{x} \leq 0.3$ the droplet is in the spherical cap regime. As shown in supplementary video $\mathrm{S} 1$ in the final stages of relaxation the droplet spreads slightly along the short axis of the rivulet (ydirection) while along the long axis (x-direction) the contact line continues to retract, this gives rise to a slightly elliptical final contact line shape ${ }^{27,28}$. It is clear from Fig. 1 that the effect of a voltage-selected $\theta_{R}$ is a change in the overall timescale of the dewetting process. Increasing the time to reach equilibrium from the order of $1000 \mathrm{~ms}$ with $V_{R}=0 \mathrm{~V}$ (Fig. 1b) to the order of $6000 \mathrm{~ms}$ with $V_{R}=180 \mathrm{~V}$ (Fig. 1d), a six-fold increase in timescale.

We note here the high degree of directionality of the dewetting behaviour observed in Fig. 1. With $V_{R}=0$ the rectangular thin film dewets both parallel and perpendicular to the underlying electrode structure. As $V_{R}$ increases dewetting in the perpendicular direction reduces being suppressed completely at high values of $V_{R}$ forcing a constant width throughout dewetting (see Fig. 1d). We attribute this to the emergence of electrostatic potential energy barriers along the edges of the electrode fingers which slow the motion and locally pin the contact line at low and high retraction voltages respectively ${ }^{9}$. For simplicity and continuity we discuss the results of the dewetting in the $\mathrm{x}$-direction only.

The dynamic contact angle as a function of time for the relaxing film under different $V_{R}$ is shown in Fig. 2a. Due to the geometry of the initial transient and rivulet we observe the appearance of the onset of the pearling instability which acts to pinch-off droplets from the ends of the rivulet ${ }^{22,29}$. The formation of a neck leads an initial rapid rise of $\theta_{d}$ as liquid is squeezed into the ends of the rivulet followed by a decrease in $\theta_{d}$ as the neck heals and liquid is redistributed into the rivulet. As the contact line movement in the y-direction is slowed and eventually suppressed by $V_{R} \neq 0 \mathrm{~V}$ we observe a reduction in 
a)

b)

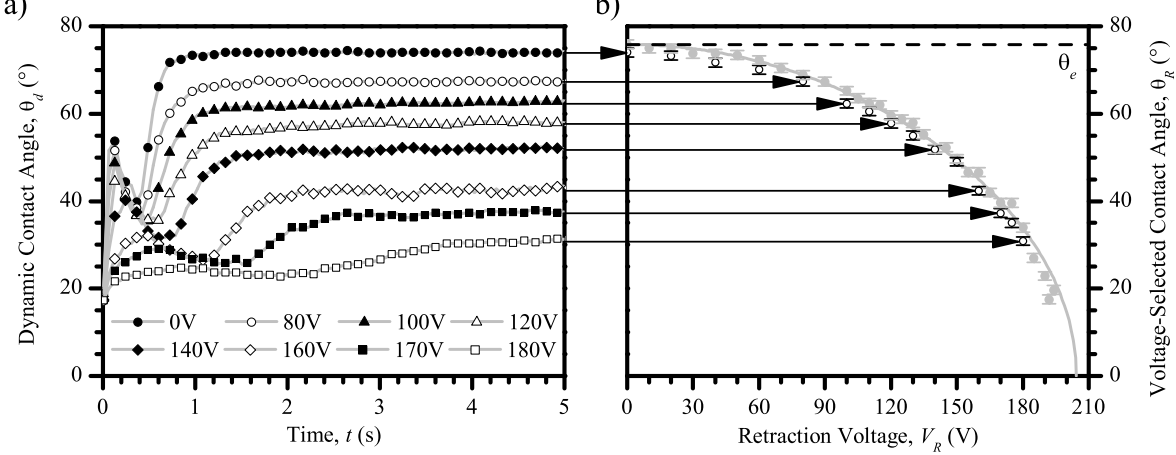

FIG. 2. a) Dynamic contact angle as a function of time whilst a constant retraction voltage $V_{R}$ is applied. For clarity we show a data point only every $120 \mathrm{~ms}$. b) Measured voltage-selected final contact angles from the dewetting experiments (hollow circles). Measurements made from experiments where the voltage is quasi-statically reduced from $V_{t h}$ (grey filled circles) are shown for comparison purposes. Grey solid line shows the fit to $\cos \theta(V)=\cos \theta_{e}+\alpha V^{2}$ using the parameters, $\alpha=1.80 \pm 0.02 \times 10^{-5} \mathrm{~V}^{-2}$ and $\theta_{e}=75.8 \pm 0.3^{\circ}$

neck formation with increasing $V_{R}$ which smooths out the initial rise and fall of the dynamic contact angle.

During the spherical cap regime we observe a switch in dewetting behaviour to an exponential relaxation towards the voltage-selected final contact angle described by $\theta_{R}-\theta_{d}=$ $\tau d \theta_{d} / d t$ where $\tau$ is the timescale of the relaxation ${ }^{4}$. We obtain quasi-static measurements of $\theta(V)$ by fully spreading to the rectangular film state and then quasi-statically reduce the applied voltage in steps of $5 \mathrm{~V}$ while measuring $\theta(V)$ in the direction parallel to the electrode stripes. We find that the voltage-selected final contact angle from the dynamic experiments correlates well with the values from the static calibration experiment made by quasi-statically reducing the applied voltage from above $V_{t h}$ (see Fig. 2b).

Fig. 3a shows the rescaled base length as a function of time. We find that after the initial transient the capillary rim regime is characterised by a linear decrease in $L$ with time, indicating that the dewetting speed $U=d L / d t$ remains constant. We measure the speed of the receding contact line after the initial transient (see solid lines in Fig. 3a) where we find $U=3.53 \mathrm{~mm} \mathrm{~s}^{-1}$ for $V_{R}=0$. The Reynolds number compares the effect of inertia and viscosity and is given by $R e=\rho U R / \eta \approx 10^{-2}$, indicating the observed dewetting remains within the viscous regime, therefore the expected scaling is $U \propto \theta_{e}^{3}$ for a dewetting capillary rim ${ }^{5}$. It is clear that as $V_{R}$ increases and $\theta_{R}$ reduces the speed of dewetting decreases. For example, $U=3.53 \mathrm{~mm} \mathrm{~s}^{-1}$ for $\theta_{R}=74.8^{\circ}$ and $U=0.42 \mathrm{~mm} \mathrm{~s}^{-1}$ for $\theta_{R}=30.9^{\circ}$ a total reduction in velocity of $88 \%$. Fig. $3 \mathrm{~b}$ shows the comparison between the measurements of $U$ at various $\theta_{R}$ to the expected scaling where we find an excellent agreement for the range of contact angles explored.

At low retraction voltages we observe an increase in $U$ by as much as $10 \%$ compared to the $0 \mathrm{~V}$ case despite a small reduction in $\theta_{R}$. As discussed above, the existence of the pearling instability at low voltages resists the motion of the contact line to dewetting in the $\mathrm{x}$-direction being prominent at $0 \mathrm{~V}$. As the onset of instability is quickly suppressed by application of a retraction voltage, this leads to an increase in dewetting speed before the final contact angle effect dominates.

In the spherical cap regime the measurements of $\tau$ are extracted from exponential fits to the $L(t)$ data by plotting a graph of $\left.\ln \left(L(t)-L_{R}\right)\right)$ against time, where a trial value of $L_{R}$ is first used which is the final measured value of $L_{R}$. We then fit a straight line to this plot between $\left(0.01<L(t)-L_{R}<0.2\right)$. We then change the value of $L_{R}$ until the correlation coefficient, $R^{2}$ is maximised (see inset in Fig. 3a). There is a clear increase in the timescale of the exponential regime with increasing retraction voltage and decreasing $\theta_{R}$. For example, $\tau=0.098 \mathrm{~s}$ at $\theta_{R}=74.8^{\circ}$ and $\tau=0.59 \mathrm{~s}$ at $\theta_{R}=30.9^{\circ}$ $\left(V / V_{t h}=0.88\right)$ a six-fold increase in relaxation time. The timescale of dewetting is expected to scale as $\tau \propto \theta_{R}^{-10 / 3}$ for a spherical cap droplet ${ }^{4}$. Comparing the measurements of $\tau$ while the final drop shape remains elliptical in nature, $V_{R}<130 \mathrm{~V}$, to the expected scaling shows an excellent agreement with range of contact angles explored (see Fig. 3c). A linear regression fit for voltages between $0 \mathrm{~V}$ and $130 \mathrm{~V}$ to the data shows the exponent as $-3.2 \pm 0.2$.

In summary, our results show that the dynamics of both dewetting regimes can be precisely controlled by a noncontact electrostatic selection of the final contact angle, $\theta_{R}$. We demonstrate a reduction in linear velocity of up to $88 \%$ and up to a six-fold increase in the timescale of the exponential regime with excellent agreement with existing theoretical predictions. We note the suppression of the pearling instability by electrostatically increasing the directionality of dewetting which for low retraction voltages leads to an increase in the speed of dewetting in the capillary rim regime. Future study of higher aspect ratio retracting rivulets would further elucidate the mechanisms of electrostatic suppression 

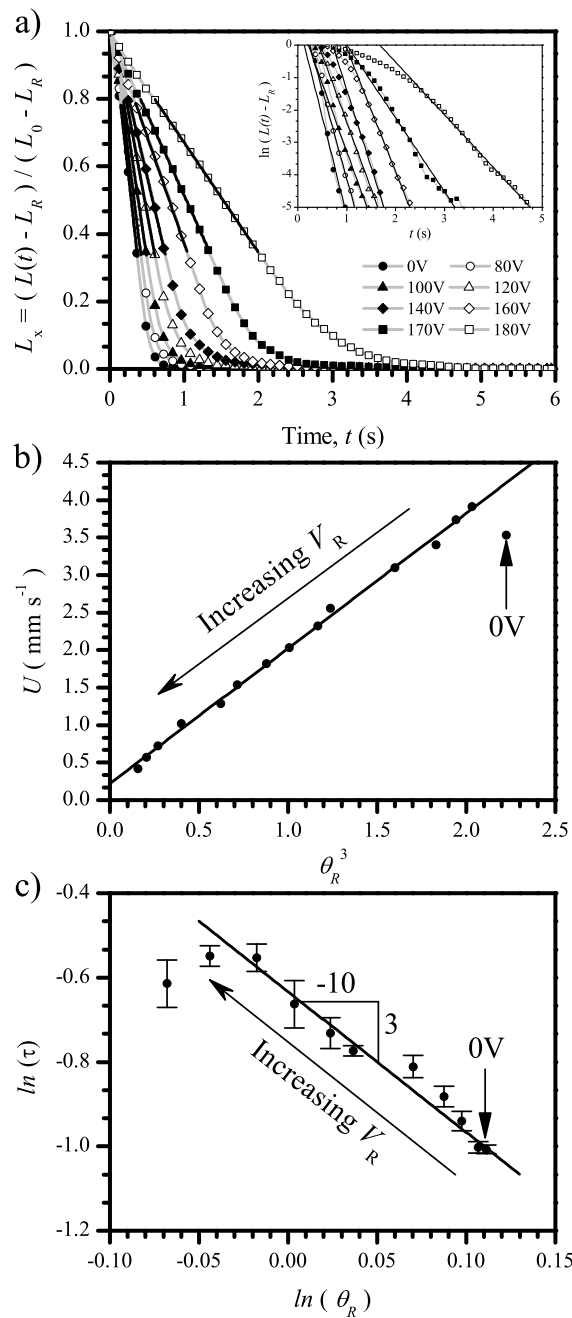

FIG. 3. a) Plot of rescaled base length as a function of time for various $V_{R}$. Inset shows exponential fitting to the plots of $\ln (L(t)-$ $\left.L_{R}\right)$. b) Comparison of the contact line speed in the capillary rim regime and the expected scaling, $U \propto \theta_{R}^{3}$. Solid lines are linear fits to the data. c) $\log -\log$ plot of the timescale of the exponential regime, $\tau$ as a function of the voltage-selected final contact angle, $\theta_{R}$ solid line is a guide to the eye showing the expected scaling $\tau \propto \theta_{R}^{-10 / 3}$.

of the pearling instability and prevention of associated droplet pinch-off. While the experiments in this work have been performed using a non-volatile liquid, the underpinning effect of liquid dielectrophoresis has been shown to work with a range of common volatile solvents over a wide range of surface tension and viscosity $11,19,30-32$. Since this approach is able to dynamically and locally modify the speed of dewetting on a surface without the need for chemical patterning providing valuable insights into printing ${ }^{33}$ and micropatterning ${ }^{1,34}$. The findings may be significant in microfluidics ${ }^{11}$ and liquid displays ${ }^{35}$.

See supplementary material for side and top views for the relaxation of a thin liquid film at three different retraction voltages (video S1). The data that support the findings of this study are available from the corresponding author upon reasonable request.

This work was supported by the U.K. Engineering and Physical Sciences Research Council grants EP/R042276/1 and EP/R036837/1.

${ }^{1}$ D. Gentili, G. Foschi, F. Valle, M. Cavallini, and F. Biscarini, "Applications of dewetting in micro and nanotechnology," Chemical Society Reviews 41, 4430-4443 (2012).

${ }^{2}$ G. Henn, D. Bucknall, M. Stamm, P. Vanhoorne, and R. Jérôme, "Chain end effects and dewetting in thin polymer films," Macromolecules 29 , 4305-4313 (1996)

${ }^{3}$ J. N. Helbert and N. Saha, "Application of silanes for promoting resist patterning layer adhesion in semiconductor manufacturing," Journal of Adhesion Science and Technology 5, 905-925 (1991).

${ }^{4}$ A. M. J. Edwards, R. Ledesma-Aguilar, M. I. Newton, C. V. Brown, and G. McHale, "Not spreading in reverse: The dewetting of a liquid film into a single drop," Science Advances 2, e1600183 (2016).

${ }^{5}$ C. Redon, F. Brochard-Wyart, and F. Rondelez, "Dynamics of dewetting," Physical Review Letters 66, 715 (1991).

${ }^{6}$ P.-G. De Gennes, F. Brochard-Wyart, and D. Quéré, Capillarity and Wetting Phenomena: Drops, Bubbles, Pearls, Waves (Springer Science \& Business Media, 2013).

${ }^{7}$ A. W. Adamson and A. P. Gast, Physical Chemistry of Surfaces, Vol. 6 (Wiley-Blackwell, 1967)

${ }^{8}$ F. Mugele and J.-C. Baret, "Electrowetting: From basics to applications," Journal of Physics: Condensed Matter 17, R705 (2005).

A. M. J. Edwards, C. V. Brown, M. I. Newton, and G. McHale, "Dielectrowetting: The past, present and future," Current Opinion in Colloid \& Interface Science 36, 28-36 (2018).

${ }^{10}$ S. K. Cho, H. Moon, and C.-J. Kim, "Creating, transporting, cutting, and merging liquid droplets by electrowetting-based actuation for digital microfluidic circuits," Journal of Microelectromechanical systems 12, 70-80 (2003).

${ }^{11}$ H. Geng, J. Feng, L. M. Stabryla, and S. K. Cho, "Dielectrowetting manipulation for digital microfluidics: Creating, transporting, splitting, and merging of droplets," Lab on a Chip 17, 1060-1068 (2017).

${ }^{12}$ J. Heikenfeld, P. Drzaic, J.-S. Yeo, and T. Koch, "A critical review of the present and future prospects for electronic paper," Journal of the Society for Information Display 19, 129-156 (2011)

${ }^{13}$ S. Xu, H. Ren, Y. Liu, and S.-T. Wu, "Color displays based on voltagestretchable liquid crystal droplet," Journal of Display Technology 8, 336340 (2012)

${ }^{14} \mathrm{~B}$. Berge and J. Peseux, "Variable focal lens controlled by an external voltage: An application of electrowetting," The European Physical Journal E 3, 159-163 (2000).

${ }^{15} \mathrm{~S}$. Xu, H. Ren, and S.-T. Wu, "Dielectrophoretically tunable optofluidic devices," Journal of Physics D: Applied Physics 46, 483001 (2013).

${ }^{16} \mathrm{H}$. Li, M. Paneru, R. Sedev, and J. Ralston, "Dynamic electrowetting and dewetting of ionic liquids at a hydrophobic solid-liquid interface," Langmuir 29, 2631-2639 (2013).

${ }^{17}$ M. Paneru, C. Priest, R. Sedev, and J. Ralston, "Static and dynamic electrowetting of an ionic liquid in a solid/liquid/liquid system," Journal of the American Chemical Society 132, 8301-8308 (2010).

${ }^{18} \mathrm{M}$. Vallet, M. Vallade, and B. Berge, "Limiting phenomena for the spreading of water on polymer films by electrowetting," The European Physical Journal B-Condensed Matter and Complex Systems 11, 583-591 (1999). 
${ }^{19}$ G. McHale, C. V. Brown, M. I. Newton, G. G. Wells, and N. Sampara, Dielectrowetting driven spreading of droplets," Physical Review Letter 107, 186101 (2011).

${ }^{20}$ G. McHale, C. V. Brown, and N. Sampara, "Voltage-induced spreading and superspreading of liquids," Nature Communications 4, 1-7 (2013).

${ }^{21}$ A. M. J. Edwards, R. Ledesma-Aguilar, M. I. Newton, C. V. Brown, and G. McHale, "A viscous switch for liquid-liquid dewetting," Communications Physics 3, 1-6 (2020).

${ }^{22}$ P. D. Ravazzoli, I. Cuellar, A. G. González, and J. A. Diez, "Wetting an dewetting processes in the axial retraction of liquid filaments," Physical Review E 95, 053111 (2017).

${ }^{23}$ C. Brown, G. G. Wells, M. I. Newton, and G. McHale, "Voltageprogrammable liquid optical interface," Nature Photonics 3, 403-405 (2009)

${ }^{24}$ J. Barman, W. Shao, B. Tang, D. Yuan, J. Groenewold, and G. Zhou, "Wettability manipulation by interface-localized liquid dielectrophoresis: Fundamentals and applications," Micromachines 10, 329 (2019).

${ }^{25}$ S. H. Davis, "Moving contact lines and rivulet instabilities. part 1. the static rivulet," Journal of Fluid Mechanics 98, 225-242 (1980).

${ }^{26}$ T. S. Chan, J. D. McGraw, T. Salez, R. Seemann, and M. Brinkmann, "Morphological evolution of microscopic dewetting droplets with slip," Journal of Fluid Mechanics 828, 271-288 (2017).

${ }^{27}$ P. D. Ravazzoli, A. G. González, and J. A. Diez, "Drops with non-circular footprints," Physics of Fluids 28, 042104 (2016)
${ }^{28}$ I. Cuellar, P. D. Ravazzoli, J. A. Diez, and A. G. González, "Drop pattern resulting from the breakup of a bidimensional grid of liquid filaments," Physics of Fluids 29, 102103 (2017).

${ }^{29}$ T. R. Powers, D. Zhang, R. E. Goldstein, and H. A. Stone, "Propagation of a topological transition: The rayleigh instability" Physics of Fluids $\mathbf{1 0}$ 1052-1057 (1998).

${ }^{30} \mathrm{R}$. Ahmed and T. B. Jones, "Dispensing picoliter droplets on substrates using dielectrophoresis," Journal of Electrostatics 64, 543-549 (2006).

${ }^{31}$ D. Chugh and K. V. Kaler, "Integrated liquid and droplet dielectrophoresis for biochemical assays," Microfluidics and Nanofluidics 8, 445-456 (2010). ${ }^{32}$ Z. Brabcova, G. McHale, G. G. Wells, C. V. Brown, and M. I. Newton, "Electric field induced reversible spreading of droplets into films on lubricant impregnted suf sce", Applied Physes Leters 110, 121603 (2017).

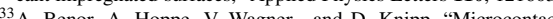
A. Benor, A. Hoppe, V. Wagner, and D. Knipp, "Microcontact printing and selective surface dewetting for large area electronic applications," Thin Solid Films 515, 7679-7682 (2007).

${ }^{34}$ M. L. Chabinyc, W. S. Wong, A. Salleo, K. E. Paul, and R. A. Street, "Organic polymeric thin-film transistors fabricated by selective dewetting," Applied Physics Letters 81, 4260-4262 (2002).

${ }^{35}$ Z. Yi, W. Feng, L. Wang, L. Liu, Y. Lin, W. He, L. Shui, C. Zhang, Z. Zhang, and G. Zhou, "Aperture ratio improvement by optimizing the voltage slope and reverse pulse in the driving waveform for electrowetting displays," Micromachines 10, 862 (2019). 

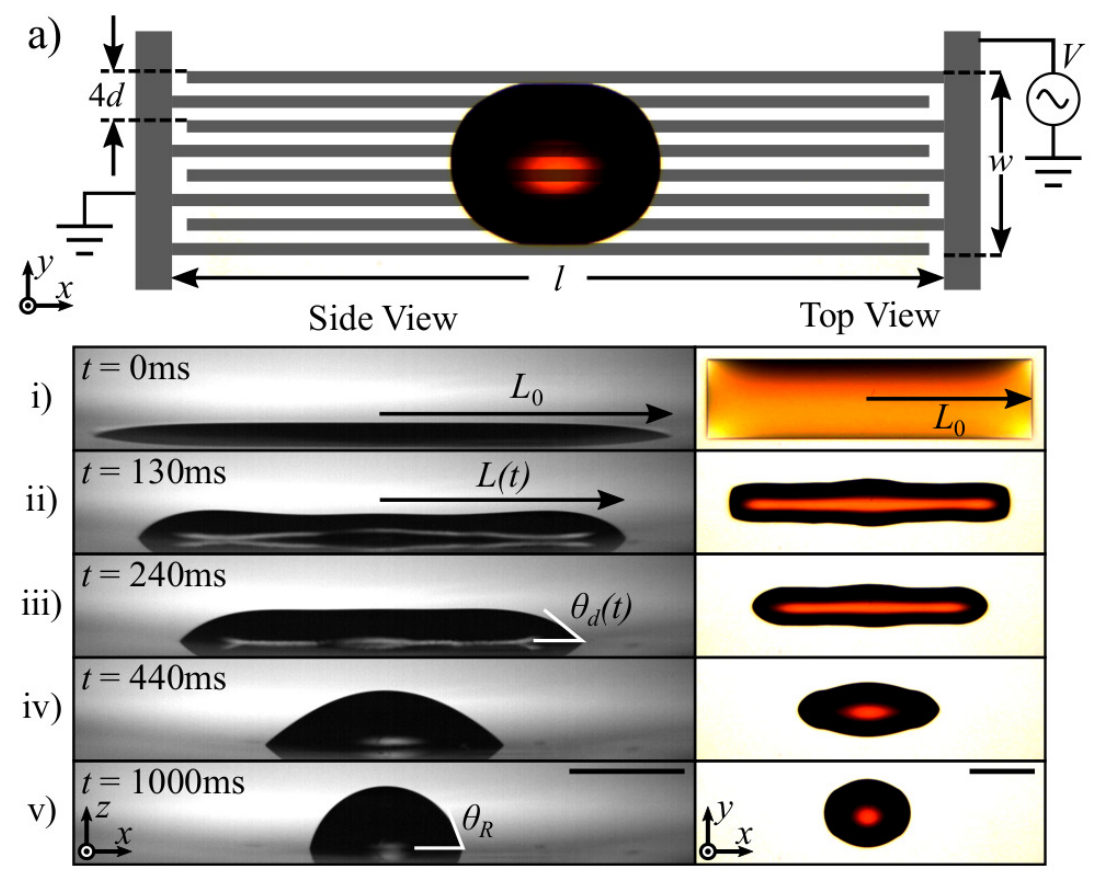

b) $\theta_{R}=74.8^{\circ}$

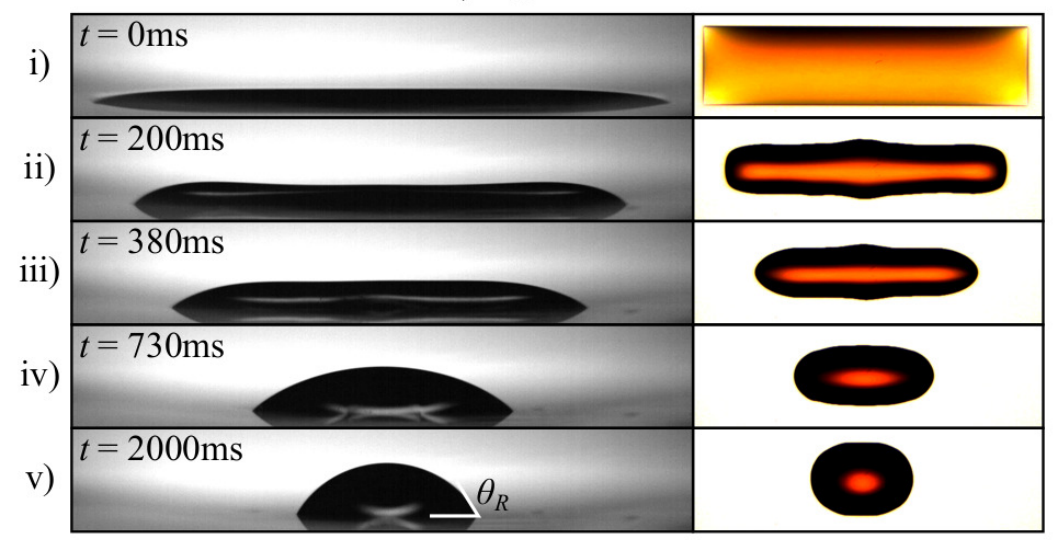

c) $\theta_{R}=59.4^{\circ}$

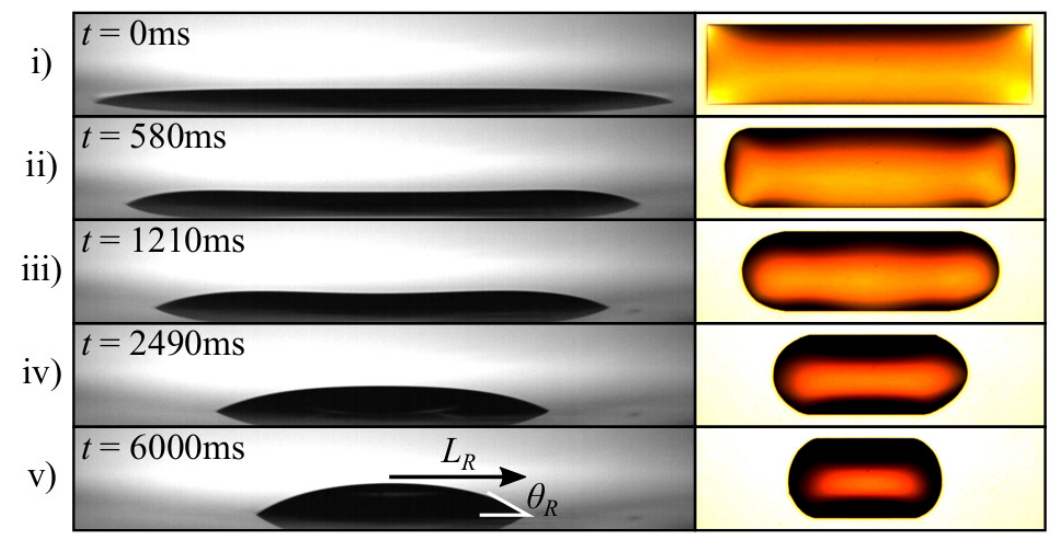

d) $\theta_{R}=30.9^{\circ}$ 


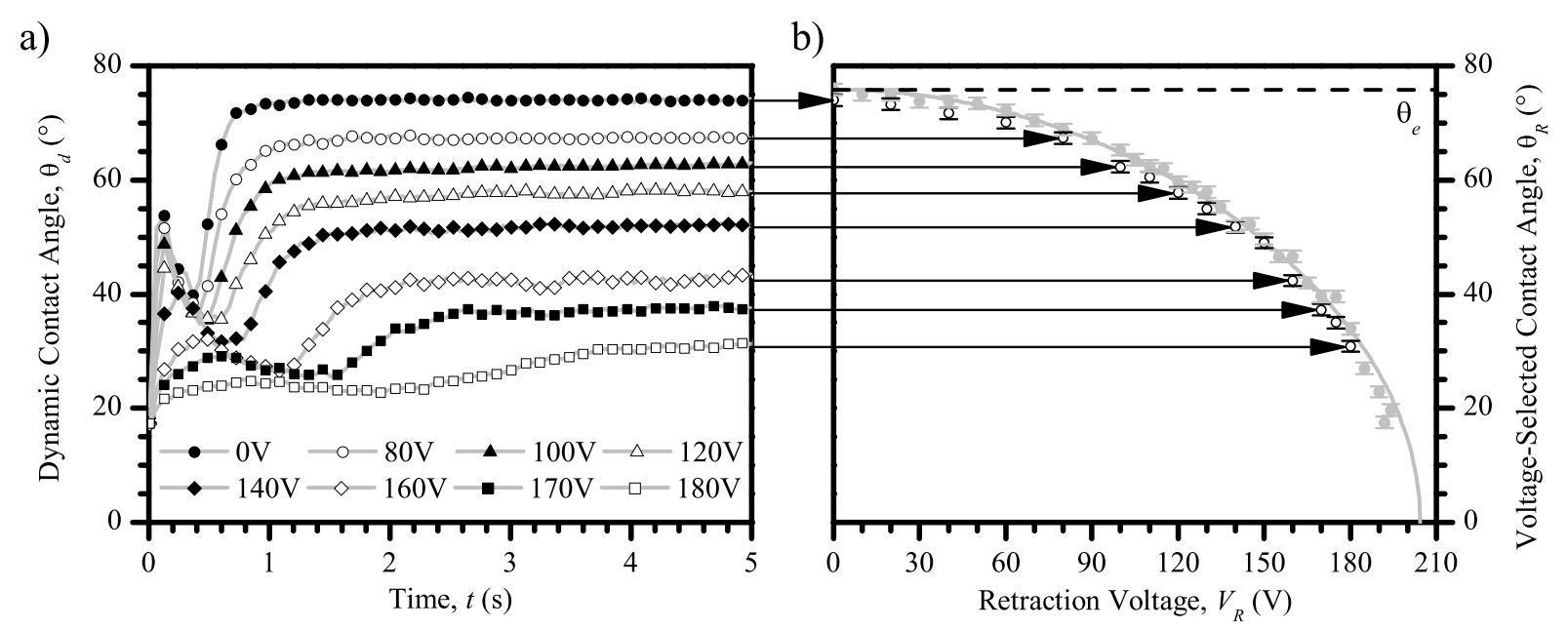



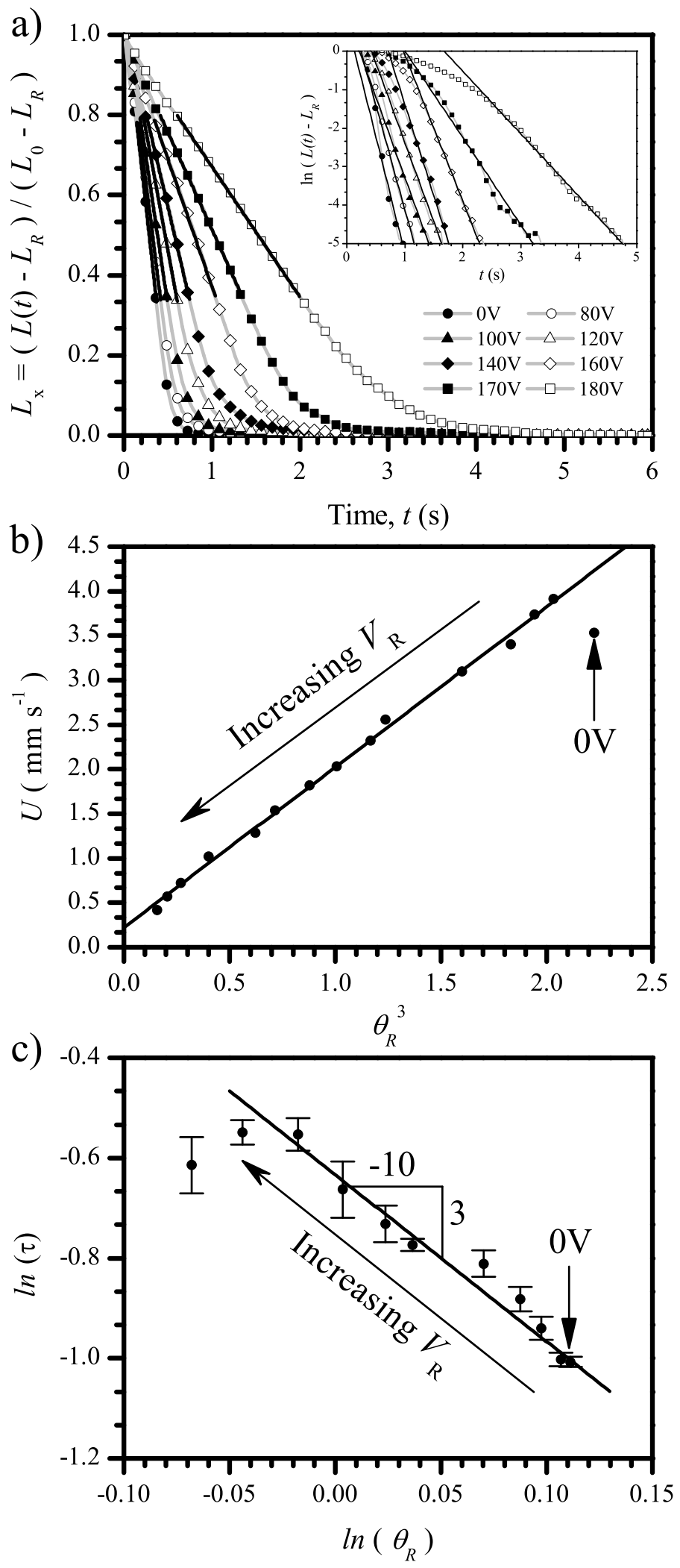